\title{
Anchoring Copper Single Atoms on Porous Boron Nitride Nanofiber to Boost Selective Reduction of Nitroaromatics
}

\section{Jianli Liang}

Center of Super-Diamond and Advanced Films (COSDAF) and Department of Chemistry, City University of Hong Kong

\section{Qianqian Song}

Department of Materials Science and Engineering, Sun Yat-Sen University

\section{Jianghua Wu}

National Laboratory of Solid State Microstructures, Jiangsu Key Laboratory of Artificial Functional Materials, College of Engineering and Applied Sciences and Collaborative Innovation Center of Adva Qi Lei

Shanghai Synchrotron Radiation Facility, Shanghai Institute of Applied Physics, Chinese Academy of Sciences

\section{Zhongming Huang}

Center of Super-Diamond and Advanced Films (COSDAF) and Department of Chemistry, City University of Hong Kong

\section{Tianxing Kang}

Center of Super-Diamond and Advanced Films (COSDAF) and Department of Chemistry, City University of Hong Kong

\section{Hui Xu}

\section{Jiangsu University}

\section{Peng Wang}

Nanjing University https://orcid.org/0000-0003-0788-6687

\section{Xingtai Zhou}

Shanghai Institute for Applied Physics

\section{Po Keung Wong}

https://orcid.org/0000-0003-3081-960X

\section{Zhifeng Jiang}

Department of Chemistry \& Center of Super-Diamond and Advanced Films (COSDAF), City University of Hong Kong, Institute for Energy Research, Jiangsu University

\section{Chun-Sing Lee ( $\sim$ apcslee@cityu.edu.hk)}

City University of Hong Kong https://orcid.org/0000-0001-6557-453X 


\section{Article}

Keywords: nanofibers, single atoms catalysts, BNNF-Cu catalyst

Posted Date: June 8th, 2021

DOl: https://doi.org/10.21203/rs.3.rs-576178/v1

License: (c) (i) This work is licensed under a Creative Commons Attribution 4.0 International License. Read Full License

Version of Record: A version of this preprint was published at ACS Nano on February 16th, 2022. See the published version at https://doi.org/10.1021/acsnano.1c10003. 


\section{Abstract}

Single atom catalysts have received widespread attention for their fascinating performance in terms of metal atom efficiency as well as their unique catalysis mechanisms comparing to conventional catalysts. Here, we prepared a high-performance catalyst of single-Cu-atom-decorated boron nitride nanofibers (BNNF-Cu) via a facile calcination method for the first time. The as-prepared catalyst shows excellent catalytic activity and good stability for converting different nitro compounds into their corresponding amines both with and without photoexcitation. By combined studies using synchrotron radiation analysis, high-resolution high-angle annular dark-field transmission electron microscopy studies and DFT calculation, dispersion and coordination of $\mathrm{Cu}$ atoms as well as their catalytic mechanisms are explored. The BNNF-Cu catalyst is found to have a record high turn-over frequency comparing to previously reported nonprecious-metal-based catalysts. While the performance of the BNNF-Cu catalyst is only of the middle range level among the state-of-the-art precious-metal-based catalysts, due to the much lower cost of the BNNF-Cu catalyst, its cost-efficiency is the highest among these catalysts. This work provides a new choice of support material which can promote the development of single atoms catalysts.

\section{Introduction}

Single atoms catalysts (SACs), as one of the most innovative and active research frontiers in the field of catalysis, have recently attracted a lot of attention due to their compelling merits such as ultra-high activity and selectivity, nearly $100 \%$ atom-utilization efficiency, easy identification of the reaction mechanisms and so on ${ }^{1-3}$. The SACs have good potential for environmental, energy-conversion and industrial utilizations that are traditional dominated by conventional metal catalysts. Different from conventional catalysts, in SACs, the interaction between the metal and the reactive species changes due to the decreased electron density caused by positively charged metal atoms. Moreover, the uniform dispersion of single metal atoms in SACs can also lead to the change of reactive intermediates adsorption mode and prevent side reactions ${ }^{4-6}$.

To date, various SACs have been synthesized via anchoring single metal atoms on numerous materials ${ }^{7-}$ 13. Isolated metal atoms are generally stabilized by surface oxo ligands ${ }^{14,15}$, defects (vacancies and monoatomic step edges) ${ }^{16-19}$, heteroatoms ${ }^{20-22}$ and confined space ${ }^{23-26}$ of supporting materials. Porous materials such as zeolites, MOFs, and COFs are considered as perfect SACs supports due to their unique pore structures, large surface areas, high defect densities which are beneficial to uniform dispersion and stabilization of isolated metal atoms. Porous boron nitride $(\mathrm{p}-\mathrm{BN})$ which possesses the advantages of porous materials and good oxidation resistance as well as excellent physical and chemical inertness ${ }^{27,28}$ has attracted wide attention and have been utilized in various fields, such as pollution treatment ${ }^{27,29}$, gases capturing and storage ${ }^{30,31}$, catalysis etc ${ }^{32,33}$. Although some theoretical works have predicted that the BN can stabilize isolated metal atoms and exhibit certain catalytic activity $^{34-37}$, there is, so far, no report on BN-based SACs. 
In this work, for the first time we successfully prepared a porous BN nanofibers/single Cu atom (BNNF$\mathrm{Cu}$ ) catalyst via a facile in-situ pyrolysis strategy. Dispersion of $\mathrm{Cu}$ single atoms and coordination between $\mathrm{BN}$ and $\mathrm{Cu}$ atoms were systematically analyzed and confirmed with synchrotron radiation analysis and high-resolution high-angle annular dark-field transmission electron microscopy. The existence of isolated $\mathrm{Cu}$ atoms on BNNF leads to significantly enhanced catalytic performance for selectively converting nitro compounds into amines. Notably, the turnover efficiency of BNNF-Cu is considerably higher than and comparable to those of previously reported nonprecious- and preciousmetal-based catalysts respectively. Furthermore, density functional theory (DFT) calculation was also carried out to explore the reaction pathway and explain the mechanism of the catalytic processes. It is believed that the successful preparation of BNNF-Cu provides a facile strategy to simultaneously achieve good dispersion of single metal atoms and high catalytic activity as well as good stability, promoting the development of SACs.

\section{Results}

Synthesis and characterization of materials. BNNF-Cu with different copper contents were prepared via a facile method using low-cost raw materials. $\mathrm{CuCl}_{2}$, melamine and boric acid were first reacted to form a gel-like BNNF-Cu precursor. BNNF-Cu was obtained by annealing the precursor at $900{ }^{\circ} \mathrm{C}$ as stated in detail in the Methods section. Catalytic activity of the BNNF-Cu was optimized by regulating the amount of copper salt added. As shown in Supplementary Fig. 1-2, the BNNF-Cu samples prepared with different copper concentrations have similar crystal structures and functional groups. Supplementary Fig. 3 shows that BNNF-Cu-2 has the highest catalytic activity. Thus, subsequent studies would be concentrated on BNNF-Cu-2 and it will be simply referred as BNNF-Cu hereafter. Figure 1a shows a schematic diagram of BNNF-Cu's preparation process.

Microstructure and morphology of BNNF-Cu were characterized with field-emission scanning electron microscopy (FE-SEM) and transmission electron microscopy (TEM). As shown in Fig. 1b-d, the asprepared sample showed a fiber-like structure with a rectangular cross-section (see Supplementary Fig. 4) of about $200 \mathrm{~nm}$ width and relatively low crystallinity (Fig. 1c, inset). There is no obvious difference between BNNF-Cu and BNNF synthesized without $\mathrm{Cu}$ (Supplementary Fig. 5), indicating that the addition of $\mathrm{Cu}^{2+}$ ions does not affect the samples' morphology. Fourier transform infra-red (FT-IR) spectra of BNNF and BNNF-Cu were shown in Supplementary Fig. 6. A spectrum from commercially purchased boron nitride nanosheets (BNNS) was also shown for comparison. All the three samples clearly show two characteristic B-N vibrations at $\sim 1380 \mathrm{~cm}^{-1}$ (B - N transverse stretching in plane) and $\sim 800 \mathrm{~cm}^{-1}(\mathrm{~B}-$ $\mathrm{N}-\mathrm{B}$ bending out of plane). There are also additional peaks in BNNF and BNNF-Cu located between 3200 $\sim 3600 \mathrm{~cm}^{-1}$ relating to functional groups $-\mathrm{OH}$ and $\mathrm{v}(\mathrm{N}-\mathrm{H})$ and a weak vibration at $1637 \mathrm{~cm}^{-1}$ probably due to $\delta(\mathrm{N}-\mathrm{H})^{28}$. The introduction of these functional groups probably leads to enhancement of adsorption capacity. The counter-phase $\mathrm{B}-\mathrm{NE}_{2 \mathrm{~g}}$ Raman vibration mode of boron nitride at $\sim 1370 \mathrm{~cm}^{-1}$ can be observed in Raman spectra of all three samples (Supplementary Fig. 7) ${ }^{38}$. The main difference is that the Raman peak is much shaper in BNNS which is highly crystalline hexagonal boron nitride (Fig. $1 \mathrm{~g}$ 
and Supplementary Fig. 8). The noisy spectra, board and shifted Raman peaks of BNNF and BNNF-Cu are due to their low crystallinity. Supplementary Fig. 9 shows X-ray photoelectron spectroscopy (XPS) survey spectra from BNNF and BNNF-Cu. Other than $B$ and $N$, signal from $O$ and $C$ are attributed to surface contamination. It is interesting to note that almost no $\mathrm{Cu}$ signal was detected even in the BNNF-Cu sample, suggesting that the amount of $\mathrm{Cu}$ in it is close to or lower than detection limit of our XPS system. The above analysis shows that adding $\mathrm{Cu}^{2+}$ in the reactant will not cause significant impact on the morphology, crystal structure nor functional groups of the formed BNNF or BNNF-Cu. We can also conclude that the amount of $\mathrm{Cu}$ in the BNNF-Cu should be low $(<1 \%)$.

We then employed more sophisticated analytical techniques including synchrotron radiation analysis, high-resolution high-angle annular dark-field (HAADF) transmission electron microscopy as well as electron paramagnetic resonance (EPR) spectroscopy to determine whether there is really copper in the BNNF-Cu sample as well as the distribution and nature of copper if it does exist. Elemental HAADF mapping of B, N, Cu in BNNF-Cu (Fig. 1e and Supplementary Fig. 10) verified the homogeneous distribution of $\mathrm{Cu}$. Importantly, many blue single spots in the element mapping image of partial BNNF-Cu and bright spots in the high-resolution high-angle annular dark-field (HAADF) image (Fig. 1f) both indicated that isolated (or few-atom clusters of) Cu atoms were successfully and evenly anchored on BNNF. X-ray diffraction (XRD) patterns of BNNF, BNNF-Cu and BNNS show two primary diffraction peaks at about $26.5^{\circ}$ and $42.5^{\circ}$, corresponding to the (002) plane and $(100) /(101)$ of hexagonal BN (PDF340421), respectively ${ }^{30}$. Interestingly, comparing the peaks of these three layered samples, the (002) peak of BNNF-Cu is slightly shift to lower angel, meaning that the interlayer distance of BNNF-Cu is expanded probably attributed to the insertion of $\mathrm{Cu}$ atoms between $\mathrm{BN}$ layers ${ }^{40}$. Furthermore, the decrease of the primary diffraction peak intensity for BNNF-Cu, in contrast to BNNF, suggests that the insertion of $\mathrm{Cu}$ atoms does distort the crystal structure of BNNF to some extent ${ }^{41}$. In addition, the specific surface area of BNNF-Cu was determined to be $153 \mathrm{~m}^{2} \mathrm{~g}^{-1}$ which is higher than that of BNNS and BNNF (Supplementary Fig. 11 and Supplementary Table 1). The improvement of specific surface area can be attributed to the existence of $\mathrm{Cu}$ atoms providing more adsorption sites and increasing the probability of pore formation.

To further investigate the dispersion of $\mathrm{Cu}$ atoms and coordination between $\mathrm{Cu}$ atoms and BNNF, synchrotron radiation X-ray absorption near-edge spectroscopy (XANES) and extended X-ray absorption fine structure (EXAFS) measurement were conducted. Three standard samples of copper foil, CuO and $\mathrm{Cu}_{2} \mathrm{O}$ were used as references. The obtained results reveal that the $\mathrm{Cu}$ species are dispersed as isolated single $\mathrm{Cu}$ atoms and stabilized by nitrogen atoms of BNNF. As shown in Fig. 2a, the absorption edge for BNNF-Cu is sandwiched between those of $\mathrm{CuO}$ and $\mathrm{Cu}_{2} \mathrm{O}$, clearly demonstrating the electron structure with positive charges in $\mathrm{Cu}^{\delta+}(1<\delta<2)^{40}$. In addition, an electron paramagnetic resonance (EPR) spectrum of BNNF-Cu shows the characteristic $\mathrm{Cu}^{2+}$ signal $\left(\mathrm{g}_{\|}=2.33, \mathrm{~g}_{\perp}=2.05\right)^{42}$ (Fig. $\left.2 \mathrm{~d}\right)$ and a XPS high resolution $\mathrm{Cu} 2 p$ spectrum of BNNF-Cu (Supplementary Fig. 12) verified the existence of $\mathrm{Cu}^{2+}$ in BNNF$\mathrm{Cu}^{40}$. Figure $2 \mathrm{~b}$ shows Fourier transform of the $\mathrm{Cu} K$-edge EXAFS of BNNF-Cu and reference samples $\mathrm{Cu}$ foil, $\mathrm{Cu}_{2} \mathrm{O}$ and $\mathrm{CuO}$. There is a main peak at $\sim 1.6 \AA$ of $\mathrm{BNNF}-\mathrm{Cu}$, which is closing to the main peak 
positions of $\mathrm{CuO}$ and $\mathrm{Cu}_{2} \mathrm{O}$, can be attributed to the $\mathrm{Cu}-\mathrm{N}$ first shell. Additionally, no obvious peak at $2.2 \AA$ (metallic Cu-Cu coordination) can be observed. These results reveal that the atomic dispersion of $\mathrm{Cu}$ atoms in BNNF-Cu. Moreover, the wavelet transforms (WT) of Cu K-edge EXAFS also present the well dispersion of $\mathrm{Cu}$ atoms in BNNF-Cu (Fig. 2c). The WT for the EXAFS signals of BNNF-Cu, $\mathrm{Cu}$ foil, $\mathrm{CuO}_{2}$, and $\mathrm{CuO}$ are achieved by using the complex wavelet developed by $\mathrm{H}$. Hunke ${ }^{43}$. The WT contour plots of BNNF-Cu shows one maximum intensity at point $\left(4 \AA^{-1}, 1.6 \AA\right)$, which can be ascribed to the Cu-N coordination, and almost no Cu-Cu signals are observed, as compared with that of $\mathrm{Cu}$ foil, $\mathrm{CuO}$, and $\mathrm{CuO}_{2}$. Structural parameters of BNNF-Cu were further extracted by fitting the Cu K-edge EXAFS spectrum using the Artemis software. Figure 2e and Supplementary Fig. 13 show optimal fitting curves in R space and K space, respectively. The optimal fitting results are attributed to the first shell of Cu-N (bond distance: $~ 2.0$ $\AA$ ) and the second shell of Cu-B (bond distance: 2.64 $\AA$ ), as shown in Supplementary Tables 2 and 3. Moreover, there is a significant difference in the coordination number $(\mathrm{CN})$ between the first shell (Cu-N: $6.26 \pm 0.97$ ) and the of second shell (Cu-B: $2.56 \pm 0.74$ ), indicating that the coordination structure around the $\mathrm{Cu}$ single atoms is very disordered, which is consistent with XRD results. According to these results, we then build a model for density functional theory (DFT) calculation with an isolated $\mathrm{Cu}$ atomic surrounded with six Cu-N as shown in Supplementary Fig. 14.

Photocatalytic activity of BNNF-Cu. Aromatic amines are important intermediates with wild usages for synthesis of many chemicals for commercial and biomedical applications. Selective catalytic reduction of nitro compounds into amines with hydrogen or hydrogen donors has been considered as an important chemical reaction in synthetic organic chemistry ${ }^{44}$. Herein, we selected the reduction of p-nitrophenol (PNP) to p-aminophenol (PAP) with borohydride to evaluate the catalytic performance of BNNF-Cu. It is known that after adding $\mathrm{NaBH}_{4}$ to a PNP solution, 4-nitrophenolate anions with strong visible absorbance at $400 \mathrm{~nm}$ will be formed (Supplementary Fig. 15), and the concentration of 4-nitrophenolate is proportional to the intensity of the absorption peak. Under irradiation, the characteristic absorption peak of PNP gradually disappears, accompanied by the appearance PAP absorption peak centered at about $300 \mathrm{~nm}$ during the catalytic reduction of PNP over BNNF-Cu (Fig. 3a). Moreover, the existence of two isosbestic points at 280 and $314 \mathrm{~nm}$ indicates complete conversion of PNP to PAP without any byproducts ${ }^{45}$. As shown in Fig. 3b, no matter under irradiation or in dark, the $C_{t} / C_{0}$ (where $C_{t}$ is the concentration of PNP at time $t$ ) value did not change in BNNS and BNNF, indicating that these two materials cannot catalyze the reduction of PNP. By contrast, the addition of BNNF-Cu caused a rapid decrease of PNP concentration whether under photoexcitation (BNNF-Cu-D-L) or in dark (BNNF-Cu-D). These results suggested that the present of copper is essential for the catalytic action of BNNF-Cu. To further confirm this point, we decorated a BNNF sample with copper nanoparticles (BNNF-Cu-NP) with sonicating a BNNF aqueous dispersion with pre-prepared copper nanoparticle of 50 to $80 \mathrm{~nm}$ diameter (Supplementary Fig. 16). Figure 3b shows that the surface decoration of copper nanoparticles onto BNNF indeed endow it with catalytic activity both under photoexcitation and in dark. On the other hand, it can also be noted that catalytic activity of the BNNF-Cu sample is much higher than that of BNNF-Cu-NP suggesting that the isolated copper atoms or few-atom clusters on BNN-Cu do have better catalytic 
performance comparing to copper nanoparticles. Since the initial amount of the $\mathrm{NaBH}_{4}$ added is far more than needed, a pseudo first-order kinetic equation has been utilized to evaluate reaction rate, written as $I n$ $\left(C_{t} / C_{0}\right)=-k t$, (where $k$ is the apparent rate constant). The calculated rate constant $(k)$ of BNNF-Cu-L (0.65 $\left.\min ^{-1}\right)$ is more than twice that of BNNF-Cu-D $\left(0.31 \mathrm{~min}^{-1}\right.$ ) (Supplementary Fig. 17a, c), indicating a good photocatalytic activity of the BNNF-Cu. To clarify the influences of visible light excitation on the catalytic performance, a $400 \mathrm{~nm}$ filter has been equipped on Xenon lamp to block ultraviolet (UV) light with wavelength below $400 \mathrm{~nm}$. As shown in Fig. 3c, without UV light, the BNNF-Cu performed a moderate catalytic activity $(k \approx 0.43$, Supplementary Fig. $17 \mathrm{~b})$, indicating that the BNNF-Cu has a certain sensitivity to visible light.

To check the stability of the catalyst, the PNP reduction experiment was repeated by adding the same amount of PNP into the used solution after complete disappearance of original PNP's absorption peak. After 5 cycles, photocatalytic activity of BNNF-Cu shows little observable degrade (Fig. 3d). Compared the XRD pattern and FTIR spectrum of BNNF-Cu after reaction with that of the as-prepared sample, the almost same results (Supplementary Fig. 18) indicates that the crystallinity and composition of the catalyst did not change during reaction. Meanwhile, there is no observable aggregation of the isolated $\mathrm{Cu}$ atoms in BNNF-Cu after use (Supplementary Fig. 19). Thus, the BNNF-Cu shows a high operation stability under photoexcitation. Moreover, the photocatalytic universality of BNNF-Cu also estimated by reducing other nitroaromatic chemicals. As shown in Fig. 3e, f, the BNNF-Cu can quickly convert o-nitrophenol, 3nitrophenol, 3-methyl-4-nitrophenol, 2-chloro-4-nitrophenol, and 2-methyl-4-nitrophenol to their corresponding reductive amino-aromatic products under irradiation. To show the excellent performance of BNNF-Cu, a comparison of the catalytic activities with that of the recently reported catalysts was made (Fig. $3 g$ ). Because the mass of metal in catalysts and the amount of PNP in solution varied largely in different reported works, the normalized turnover frequency (TOF) was employed to evaluate the catalytic activity. As shown in Fig. 3, BNNF-Cu shows a record high TOF comparing to previously reported nonprecious-metal-based catalysts. (left panel of Fig. $3 \mathrm{~g}$ ) and even higher than many precious-metal-based catalysts (right panel of Fig. 3g). Although the TOF of BNNF-Cu is still lower than a few precious-metalbased catalysts, considering the price of copper which is much lower than precious metal (Au, Ag, Pt and Pd) (Cost per TOF are shown in Supplementary Table 4), the BNNF-Cu does possess higher costefficiency. All the above-mentioned excellent performance indicated that the BNNF-Cu catalyst has potential application prospects in the field of nitro reduction synthesis of amines.

Mechanism of enhanced catalytic performance. To further explain the positive effect of $\mathrm{Cu}$ single atoms in BNNF-Cu on catalytic activity, we systematically analyzed the band structure and electrical conductivity of BNNF-Cu. Compared with BNNF, the BNNF-Cu sample have a narrower bandgap, higher valence band position and broader light absorption range (Supplementary Fig. 20-22), revealing that the introduction of a small amount of $\mathrm{Cu}$ single atoms can change the band positions and improve light absorption capacity. Figure 4a shows the time-resolved photoluminescence (TRPL) spectroscopy of BNNF and BNNF-Cu. The PL decay lifetime ( $\tau$ ) of BNNF-Cu (2.40 ns) is slightly shorter than that of BNNF (2.73 ns), indicating an enhancement of charge transfer and separation in the BNNF-Cu system, which are probably 
ascribed to narrower bandgap and new transfer channels caused by anchoring of isolated $\mathrm{Cu}$ atoms ${ }^{10,40,46}$. Higher photocurrent density (Fig. 4b) and lower impedance of BNNF-Cu (Fig. 4c) also support the conclusion that the efficiencies of charge separation and transfer are improved by the induced isolated $\mathrm{Cu}$ atoms. As a result, anchoring small amount of isolated $\mathrm{Cu}$ atoms on BNNF can simultaneously adjust band structure and enhance the separation and transfer of electron-hole pairs.

DFT calculations were applied to gain a deeper understanding of the excellent catalytic performance of BNNF-Cu in the reduction of PNP to PAP. According to some previous works and our calculations, the configurations of adsorbate on BNNF-Cu were set to be parallel to the catalyst surface ${ }^{47,48}$. Figure 5 shows schematic diagram of the distal pathway for reduction of PNP to PAP catalyzed by BNNF-Cu and comparison of the free-energy diagrams of the reduction of PNP. In detail, after PNP adsorbed on BNNF$\mathrm{Cu}(\triangle \mathrm{E}=-1.19 \mathrm{eV})$, the first hydrogenation reaction happens that the oxygen atom of PNP combines with a hydrogen atom to form a $* \mathrm{NOOH}$ intermediate $(\triangle \mathrm{E}=-0.89 \mathrm{eV})$. Subsequently, the $* \mathrm{NOOH}$ is dehydroxylated to $* \mathrm{NO}(\triangle \mathrm{E}=-0.20 \mathrm{eV})$, followed by a second hydrogeneration that the $\mathrm{H}$ atom connected the $\mathrm{N}$ atom or $\mathrm{O}$ atom forming $* \mathrm{NHO}(\Delta \mathrm{E}=-0.85 \mathrm{eV})$ or ${ }^{*} \mathrm{NOH}(\Delta \mathrm{E}=-0.70 \mathrm{eV})$ intermediates, separately. After that, a hydrolysis reaction occurs and a new intermediate ( ${ }^{*} \mathrm{NHOH}, \Delta \mathrm{E}=-0.82 \mathrm{eV}$ or $-0.97 \mathrm{eV}$ ) is formed, which is caused by the ${ }^{*} \mathrm{NHO}$ or ${ }^{*} \mathrm{NOH}$ groups with strong Brønsted acidity. Then, the ${ }^{*} \mathrm{NHOH}$ is dehydroxylated to $* \mathrm{NH}(\triangle \mathrm{E}=-1.16 \mathrm{eV})$ before the formation of the final product of PAP $(\triangle \mathrm{E}=-1.23 \mathrm{eV})$ by the third hydrogenation. The hydrogenation reduction of PNP to PAP follows the reaction pathway that PNP $\left({ }^{\star} \mathrm{NO}_{2}\right) \rightarrow{ }^{*} \mathrm{NOOH} \rightarrow{ }^{\star} \mathrm{NO} \rightarrow{ }^{*} \mathrm{NOH}\left({ }^{*} \mathrm{NHO}\right) \rightarrow{ }^{*} \mathrm{NHOH} \rightarrow{ }^{\star} \mathrm{NH} \rightarrow$ PAP $\left({ }^{\star} \mathrm{NH}_{2}\right)$ in our BNNF-Cu system. There is no energy barrier for the hydrogenation of PNP for BNNF-Cu in the reaction pathway, in contrast, there is a large energy barrier for PNP $\left({ }^{\star} \mathrm{NO}_{2}\right) \rightarrow{ }^{\star} \mathrm{NOOH}$ for BNNF (Supplementary Fig. 23), indicating that anchoring isolated $\mathrm{Cu}$ atom on BNNF makes it suitable for reduction reactions.

\section{Discussion}

Via analysis of both the theoretical and the experimental results, the important role of the isolated $\mathrm{Cu}$ atoms in BNNF-Cu for the catalytic reduction reaction of PNP can be described as follow:

The isolated $\mathrm{Cu}$ atoms on BNNF-Cu can provide a high density of active sites for catalytic reduction of PNP to PAP. Moreover, the introduction of isolated $\mathrm{Cu}$ atoms can also improve the adsorption capacity of BNNF-Cu, making it easier for PNP molecules to be absorbed onto the catalyst surface. Under irradiation, BNNF-Cu shows an enhanced catalytic activity which is probably ascribed to the assistant of the generated photoelectrons of catalyst. In detail, when irradiating the reaction system, the catalyst will be activated, electrons are excited from the valence band (VB) to conduction band (CB) followed an easy electron transfer from $\mathrm{CB}$ to $\mathrm{Cu}$ single atoms (the Fermi level of $\mathrm{Cu}$ is commonly lower than the $\mathrm{CB}$ of BNNF), more electrons will take part in the hydrogeneration steps, thus speeding up the reaction.

In summary, we firstly reported a single atom catalyst, Cu single atoms supported by porous BN nanofibers, which is prepared by a facile in situ pyrolysis strategy. The dispersion of $\mathrm{Cu}$ single atoms and coordination between BN and Cu atoms were firstly confirmed with STEM, XAFS and other characteristic 
methods. Due to the uniform dispersion of $\mathrm{Cu}$ atoms, the as-prepared sample processes higher specific surface area, stronger light absorption capacity, faster charge transfer and easier charge separation, leading to an excellent performance in reduction of PNP with $\mathrm{NaBH}_{4}$. The good universality and stability of BNNF-Cu catalyst provide potential application in the field of catalytic reduction. Theoretical simulation systematically illustrated the pathway of the reduction of PNP, and further proved the benefits of introducing a single atom. This work not only synthesizes an efficient single atom catalyst for the photocatalytic reduction, but also provide the possibility for the application of boron nitride in field of single atom photocatalyst.

\section{Methods}

Materials. Melamine, boric acid, copper chloride dihydrate and sodium borohydride were purchased from Sigma Aldrich. P-nitrophenol, o-nitrophenol, 3-nitrophenol, 2-methyl-4-nitrophenol, 2-chloro-4-nitrophenol, 2-chloro-4-nitrophenol and boron nitride nanosheets were purchased from Aladdin chemical reagent Corp (Shanghai, China). All the chemicals were used as received.

Synthesis of BNNF-Cu and BNNF. In a typical synthesis, $0.63 \mathrm{~g}$ melamine powder and $0.618 \mathrm{~g}$ boric acid were added and dissolved into $45 \mathrm{~mL}$ deionized water via continuous stirring and heating at $80^{\circ} \mathrm{C}$ for about 30 minutes. Copper chloride solution of different concentrations ( 6.7 to $20.1 \mathrm{mg} \mathrm{CuCl}{ }_{2} \cdot 2 \mathrm{H}_{2} \mathrm{O}$ in 5 $\mathrm{mL}$ deionized water) were then respectively added dropwise into the melamine \& boric acid solution under stirring. Upon cooling to room temperature, the $\mathrm{Cu}^{2+}$ cations would form complex with melamine molecule giving gel-like precursors (melamine diborate, $M \cdot B_{2}$ ) with different copper contents. The gels were then further cooled down to $\sim-20{ }^{\circ} \mathrm{C}$ in a refrigerator. BNNF-Cu precursors were obtained by freezedrying of the cooled jellies. Subsequently, the precursors were respectively placed into a quartz tube in a furnace. The tube was first evacuated with a mechanical pump to a vacuum of $\sim 10^{-3}$ Torr. High purity $\mathrm{N}_{2}(>99.99 \%)$ was then feed into the tube at a rate of $100 \mathrm{ml} / \mathrm{min}$ with the mechanical pump running continuously. The tube was then annealed at $900^{\circ} \mathrm{C}$ for $4 \mathrm{~h}$ with the $\mathrm{N}_{2}$ feed and pumping. After naturally cooling to RT, the BNNF-Cu samples, BNNF-Cu-1, BNNF-Cu-1.5, BNNF-Cu-2, BNNF-Cu-2.5 and BNNF-Cu-3 (for reactant solutions with respectively $6.7,10.05,13.4$, and $16.75,20.1 \mathrm{mg}$ of $\mathrm{CuCl}_{2}$ ) were obtained. BNNF was synthesized via the same method without adding the copper chloride solution.

Characterizations. X-ray powder diffraction (XRD) patterns were recorded on a Bruker D2 phaser with $\mathrm{Cu}$ $\mathrm{Ka}$ radiation at $30 \mathrm{kV}$. Fourier-transform infrared (FT-IR) spectra were measured with a Nicolet iS50 system using a $\mathrm{KBr}$ disk method. Scanning electron microscopy (SEM) were carried out with a QUATTRO $S$ field-emission scanning electron microscope. Raman spectra were recorded on Renishaw inVia Raman spectrometer excited with a $633 \mathrm{~nm}$ laser. UV-vis adsorption spectra for solutions and solids were recorded with a Shimadzu 1700 and a Shimadzu solidSpec-3700/3700DUV systems, respectively. X-ray photoelectron spectroscopy (XPS) measurements were performed on Escalab 250Xi spectrometer with an Al Ka source. Photoluminance spectra and time-resolved fluorescence decay spectra were recorded with Spectrofluorometer (Edinburgh FL980). Electron paramagnetic resonance (EPR) spectra were tested with 
a Bruker A300-10/12 EPR spectrometer (center field: 3510.00 G; microwave frequency: $9.85 \mathrm{GHz}$; and microwave power: $1.87 \mathrm{~mW}$ ) at room temperature. Nitrogen adsorption-desorption measurements were performed on an automatic gas adsorption analyzer AutoSorb iQ. The Brunauer-Emmett-Teller (BET) method and the Barrett-Joyner-Halenda (BJH) method were applied for determination of specific surface area and pore size distribution, respectively. Atomic resolution scanning transmission electron microscope (STEM) observation was carried out on a double aberration-corrected FEI Titan G2 60-300 S/TEM. High angle annular dark field (HAADF) and energy dispersive X-ray spectroscopy (EDS) mapping were performed at an accelerating voltage of $300 \mathrm{kV}$. Photoelectrochemical measurements were carried out on a CHI 660B electrochemical system (Chenhua Instruments) using a conventional three-electrode cell with $\mathrm{Pt}$ as the counter electrode, and $\mathrm{Ag} / \mathrm{AgCl} / \mathrm{KCl}$ electrode as reference electrode. Transient photocurrent responses were measured under intermittent full spectrum light irradiation or intermittent visible light irradiation. Electrochemical impedance spectroscopy experiments were performed in dark.

XAFS analysis. X-ray absorption fine structure (XAFS) spectra at Cu K $(E 0=8979 \mathrm{eV})$ edge was measured at the BL14W1 beam line of the Shanghai Synchrotron Radiation Facility (SSRF). We calibrated the energy according to the absorption edge of a pure $\mathrm{Cu}$ foil. Data extraction and analysis were carried out with respectively Athena and Artemis $\operatorname{codes}^{49}$. Based on normalized X-ray absorption near edge spectroscopy (XANES) profiles, with the help of bulk references $\left(\mathrm{Cu}_{2} \mathrm{O}\right.$ for $\mathrm{Cu}^{+}$and $\mathrm{CuO}$ for $\left.\mathrm{Cu}^{2+}\right)$, oxidation state of copper can be determined by the linear combination fit referencing to bulk references $\left(\mathrm{Cu}_{2} \mathrm{O}\right.$ for $\mathrm{Cu}^{+}$and $\mathrm{CuO}$ for $\left.\mathrm{Cu}^{2+}\right)$. For the extended X-ray absorption fine structure (EXAFS) part, the Fourier transformed (FT) data in R space were analyzed by applying BN-like model with replaced $\mathrm{Cu}$ center for $\mathrm{Cu}-\mathrm{N} / \mathrm{Cu}-\mathrm{B}$. The parameters describing the electronic properties (e.g., correction to the photoelectron energy origin, E0) and local structure environment including coordination number (CN), bond distance $(\mathrm{R})$ and Debye-Waller factor around the absorbing atoms were allowed to vary during the fitting process.

\section{Calculation details}

The Vienna ab initio simulation package (VASP) ${ }^{50}$ was implemented to evaluate the reduction pathway of PNP to PAP. To model the porous BN with single $\mathrm{Cu}$ atom, two boron atoms and two nitride atoms were removed from a periodic supercell $(5 \times 5)$ of $\mathrm{BN}$ nanosheet to provide anchoring sites for a single $\mathrm{Cu}$ atom. A vacuum of $20 \AA$ along the $z$-direction was considered to avoid interaction between the periodically repeated layers. The calculations were performed at the Perdew-Burke-Ernzerhof (PBE) exchange-correlation functional within a generalized gradient approximations $(G G A)^{51,52}$. The van der Waals interactions were described using the empirical correction method (DFT-D3). The cut-off energy was set to be $500 \mathrm{eV}$. The force and energy convergence criterion were set to $0.01 \mathrm{eV} / \AA$ and $10-5 \mathrm{eV}$, respectively. A Monkhorst-Pack k-point mesh $3 \times 3 \times 1$ was used for structural optimization ${ }^{53}$. The selection of these computational parameters was based on convergence tests with respect to the total energy of the systems. 
Photocatalytic test. Photocatalytic reduction of PNP over as-prepared samples were carried out under irradiation (full spectrum light or visible light) at ambient temperature. In detail, adding $10 \mathrm{mg}$ catalysts in $80 \mathrm{~mL}$ PNP solution (10 ppm) followed 1 hour stirring in dark. Then, $11 \mathrm{mg} \mathrm{NaBH}$ was added into the solution. Keep stirring and irradiating the reaction solution during the reaction process. At a certain interval, about $2 \mathrm{~mL}$ of the reaction solution was taken out and filtered and characterized using a UV-vis absorption spectrophotometer. The photocatalytic reduction of other nitroaromatic chemicals were tested by the same method.

\section{Declarations}

\section{Data availability}

The data that support the plots within the paper and other findings of this study are available from the corresponding author upon reasonable request.

\section{Acknowledgements}

This work was supported by Hong Kong Innovation and Technology Commission (Project No. ITS/219/19) and City University of Hong Kong (ARG-CityU Applied Research Grant: Project No.9667160 and 9667179). We also thank the funding support from National Natural Science Foundation of China (Grant No. 21706102) and Scientific Research Startup of Jiangsu University (No. 202096). DFT calculation in this work was carried out using the computational facilities, CityU Burgundy, managed and provided by the Computing Services Centre at City University of Hong Kong.

\section{Author contributions}

J.L.L, Z.F.J., and C.S.L. conceived and designed the experiment. J.L.L. conducted the catalysts preparation, characterization and the catalysts testing experiments. Q.Q.S. conducted and analyzed the DFT calculation and performed the gas adsorption measurement and analysis. J.H.W. and P.W. performed the TEM characterization. Z.M.H. and T.X.K. and J.L.L. performed the Raman, FTIR and SEM tests. Q.L., X.T.Z. tested and analyzed the EXAFS data. H.X. conducted EPR measurement. J.L.L. wrote the paper, P.K.W., Z.F.J. and C.S.L revised the paper.

\section{Additional information}

Supplementary Information accompanies this paper at xxxx.

Competing interests: The authors declare no competing interests.

\section{References}

1. Wang, A., Li, J. \& Zhang, T. Heterogeneous single-atom catalysis. Nat. Rev. Chem. 2, 65-81 (2018). 
2. Cui, X., Li, W., Ryabchuk, P., Junge, K. \& Beller, M. Bridging homogeneous and heterogeneous catalysis by heterogeneous single-metal-site catalysts. Nat. Catal. 1, 385-397 (2018).

3. Zhang, H. B., Cheng, W. R., Luan,D. Y., Lou, X.-W. (David). Atomically dispersed reactive centers for electrocatalytic $\mathrm{CO}_{2}$ reduction and water splitting. Angew. Chemie Int. Ed. 2-22 (2020).

4. Guo, X. et al. Direct, nonoxidative conversion of methane to ethylene, aromatics, and hydrogen. Science. 344, 616-619 (2014).

5. Yang, $\mathrm{H}$. Bin et al. Atomically dispersed $\mathrm{Ni}(\mathrm{i})$ as the active site for electrochemical $\mathrm{CO}_{2}$ reduction. Nat. Energy 3, 140-147 (2018).

6. Li, Z. et al. Iridium single-atom catalyst on nitrogen-doped carbon for formic acid oxidation synthesized using a general host-guest strategy. Nat. Chem. 12, 764-772 (2020).

7. Sun, Q. et al. Zeolite-encaged single-atom rhodium catalysts: highly-efficient hydrogen generation and shape-selective tandem hydrogenation of nitroarenes. Angew. Chemie - Int. Ed. 58, 1857018576 (2019).

8. Wang, B., Cai, H. \& Shen, S. Single metal atom photocatalysis. Small Methods 3, 1-14 (2019).

9. Li, J. et al. Single-atom Pt- $\mathrm{N}_{3}$ sites on the stable covalent triazine framework nanosheets for photocatalytic $\mathrm{N}_{2}$ fixation. ACS Catal. 10, 2431-2442 (2020).

10. Jiang, X. H. et al. Silver single atom in carbon nitride catalyst for highly efficient photocatalytic hydrogen evolution. Angew. Chemie - Int. Ed. 59, 23112-23116 (2020).

11. Gao, C. et al. Heterogeneous single-atom photocatalysts: Fundamentals and applications. Chem. Rev. 120, 12175-12216 (2020).

12. Tiburcio, E. et al. Soluble/MOF-supported palladium single atoms catalyze the ligand-, additive-, and solvent-free aerobic oxidation of benzyl alcohols to benzoic acids. J. Am. Chem. Soc. 143, 2581-2592 (2021).

13. Zhang, $\mathrm{H}$. et al. Isolated cobalt centers on $\mathrm{W}_{18} \mathrm{O}_{49}$ nanowires perform as a reaction switch for efficient $\mathrm{CO}_{2}$ photoreduction. J. Am. Chem. Soc. 143, 2173-2177 (2021).

14. Kuai, L. et al. Titania supported synergistic palladium single atoms and nanoparticles for room temperature ketone and aldehydes hydrogenation. Nat. Commun. 11, 1-9 (2020).

15. Wang, Q. et al. Ultrahigh-loading of Ir single atoms on NiO matrix to dramatically enhance oxygen evolution reaction. J. Am. Chem. Soc. 142, 7425-7433 (2020).

16. Jones, J. et al. Thermally stable single-atom platinum-on-ceria catalysts via atom trapping. Science. 353, 150-154 (2016).

17. Wan, J. et al. Defect effects on $\mathrm{TiO}_{2}$ nanosheets: stabilizing single atomic site Au and promoting catalytic properties. Adv. Mater. 30, 1-8 (2018).

18. Ma, X. L., Liu, J. C., Xiao, H. \& Li, J. Surface single-cluster catalyst for $\mathrm{N}_{2}$-to- $\mathrm{NH}_{3}$ thermal conversion. J. Am. Chem. Soc. 140, 46-49 (2018). 
19. Liu, G. et al. $\mathrm{MoS}_{2}$ monolayer catalyst doped with isolated Co atoms for the hydrodeoxygenation reaction. Nat. Chem. 9, 810-816 (2017).

20. Li, Y., Li, B., Zhang, D., Cheng, L. \& Xiang, Q. Crystalline carbon nitride supported copper single atoms for photocatalytic $\mathrm{CO}_{2}$ reduction with nearly $100 \%$ CO selectivity. ACS Nano 14, 10552-10561 (2020).

21. Chen, P. et al. Rare-earth single-atom La-N charge-transfer bridge on carbon nitride for highly efficient and selective photocatalytic $\mathrm{CO}_{2}$ reduction. ACS Nano 14, 15841-15852 (2020).

22. Wang, Y. et al. High-efficiency oxygen reduction to hydrogen peroxide catalyzed by nickel single-atom catalysts with tetradentate $\mathrm{N}_{2} \mathrm{O}_{2}$ coordination in a three-phase flow cell. Angew. Chemie - Int. Ed. 59, 13057-13062 (2020).

23. Zuo, Q. et al. Ultrathin metal-organic framework nanosheets with ultrahigh loading of single Pt atoms for efficient visible-light-driven photocatalytic $\mathrm{H}_{2}$ evolution. Angew. Chemie - Int. Ed. 58, 10198-10203 (2019).

24. Ji, P. et al. Titanium(III)-oxo clusters in a metal-organic framework support single-site Co(II)-hydride catalysts for arene hydrogenation. J. Am. Chem. Soc. 140, 433-440 (2018).

25. Li, X. et al. Single-atom Pt as Co-catalyst for enhanced photocatalytic $\mathrm{H}_{2}$ evolution. Adv. Mater. 28, 2427-2431 (2016).

26. Chen, Z. et al. Single-site Aul catalyst for silane oxidation with water. Adv. Mater. 30, 1-8 (2018).

27. Lei, W., Portehault, D., Liu, D., Qin, S. \& Chen, Y. Porous boron nitride nanosheets for effective water cleaning. Nat. Commun. 4, 1-7 (2013).

28. Liang, J. et al. Pore structure regulation and carbon dioxide adsorption capacity improvement on porous BN fibers: Effects of high-temperature treatments in gaseous ambient. Chem. Eng. J. 373, 616-623 (2019).

29. Song, Q. et al. Nickel (II) modified porous boron nitride: An effective adsorbent for tetracycline removal from aqueous solution. Chem. Eng. J. 394, 124985 (2020).

30. Liang, J. et al. In situ Cu-loaded porous boron nitride nanofiber as an efficient adsorbent for $\mathrm{CO}_{2}$ capture. ACS Sustain. Chem. Eng. 8, 7454-7462 (2020).

31. Marchesini, S., McGilvery, C. M., Bailey, J. \& Petit, C. Template-free synthesis of highly porous boron nitride: Insights into pore network design and impact on gas sorption. ACS Nano 11, 10003-10011 (2017).

32. Liu, H. et al. Conductive boron nitride as promising catalyst support for the oxygen evolution reaction. Adv. Energy Mater. 10, 1-7 (2020).

33. Liu, Y. R. et al. Highly active Pt/BN catalysts for propane combustion: The roles of support and reactant-induced evolution of active sites. ACS Catal. 9, 1472-1481 (2019).

34. Tang, S. et al. Realizing a not-strong-not-weak polarization electric field in single-atom catalysts sandwiched by boron nitride and graphene sheets for efficient nitrogen fixation. J. Am. Chem. Soc. 142, 19308-19315 (2020). 
35. Zhao, J. \& Chen, Z. Single Mo atom supported on defective boron nitride monolayer as an efficient electrocatalyst for nitrogen fixation: A computational study. J. Am. Chem. Soc. 139, 12480-12487 (2017).

36. Lu, Z. et al. CO oxidation catalyzed by the single Co atom embedded hexagonal boron nitride nanosheet: A DFT-D study. Phys. Chem. Chem. Phys. 18, 21865-21870 (2016).

37. Deng, C., He, R., Shen, W. \& Li, M. Theoretical analysis of oxygen reduction reaction activity on single metal ( $\mathrm{Ni}, \mathrm{Pd}, \mathrm{Pt}, \mathrm{Cu}, \mathrm{Ag}, \mathrm{Au})$ atom supported on defective two-dimensional boron nitride materials. Phys. Chem. Chem. Phys. 21, 18589-18594 (2019).

38. Shankar, R. et al. Porous boron nitride for combined $\mathrm{CO}_{2}$ capture and photoreduction. J. Mater. Chem. A 7, 23931-23940 (2019).

39. Zhao, Y. et al. Can boron and nitrogen co-doping improve oxygen reduction reaction activity of carbon nanotubes? J. Am. Chem. Soc. 135, 1201-1204 (2013).

40. Xiao, X. et al. A promoted charge separation/transfer system from $\mathrm{Cu}$ single atoms and $\mathrm{C}_{3} \mathrm{~N}_{4}$ layers for efficient photocatalysis. Adv. Mater. 32, 1-8 (2020).

41. Zhang, T. et al. Preassembly strategy to fabricate porous hollow carbonitride spheres inlaid with single Cu- $\mathrm{N}_{3}$ sites for selective oxidation of benzene to phenol. J. Am. Chem. Soc. 140, 16936-16940 (2018).

42. Lee, B. H. et al. Reversible and cooperative photoactivation of single-atom $\mathrm{Cu} / \mathrm{TiO}_{2}$ photocatalysts. Nat. Mater. 18, 620-626 (2019).

43. Funke, H., Scheinost, A. C. \& Chukalina, M. Wavelet analysis of extended x-ray absorption fine structure data. 1-7 (2005) doi:10.1103/PhysRevB.71.094110.

44. Zhou, P. et al. High performance of a cobalt-nitrogen complex for the reduction and reductive coupling of nitro compounds into amines and their derivatives. Sci. Adv. 3, 1-11 (2017).

45. Hervés, P. et al. Catalysis by metallic nanoparticles in aqueous solution: Model reactions. Chem. Soc. Rev. 41, 5577-5587 (2012).

46. Xiong, X. et al. Photocatalytic $\mathrm{CO}_{2}$ reduction to $\mathrm{CO}$ over $\mathrm{Ni}$ single atoms supported on defect-rich zirconia. Adv. Energy Mater. 10, 1-8 (2020).

47. Tian, S. et al. Single-atom Fe with $\mathrm{Fe}_{1} \mathrm{~N}_{3}$ structure showing superior performances for both hydrogenation and transfer hydrogenation of nitrobenzene. Sci. China Mater. 64, 642-650 (2021).

48. Mandeep, Sharma, L. \& Kakkar, R. DFT study on the adsorption of p-nitrophenol over vacancy and Ptdoped graphene sheets. Comput. Theor. Chem. 1142, 88-96 (2018).

49. Ravel, B. \& Newville, M. ATHENA, ARTEMIS, HEPHAESTUS: Data analysis for X-ray absorption spectroscopy using IFEFFIT. J. Synchrotron Radiat. 12, 537-541 (2005).

50. Hafner J. Ab-initio simulations of materials using VASP: density-functional theory and beyond, Comput. Chem. 29, 2044-2078 (2008).

51. Perdew J.P., Wang Y. Accurate and simple analytic representation of the electron- gas correlation energy, Rev. B 45, 13244 (1992). 
52. Perdew J.P., Burke K., Wang Y. Generalized gradient approximation for the exchange-correlation hole of a many-electron system, Rev. B 54, 16533 (1996).

53. Monkhorst H.J., Pack J.D. Special points for Brillouin-zone integrations, Rev. B13, 5188 (1976).

\section{Figures}
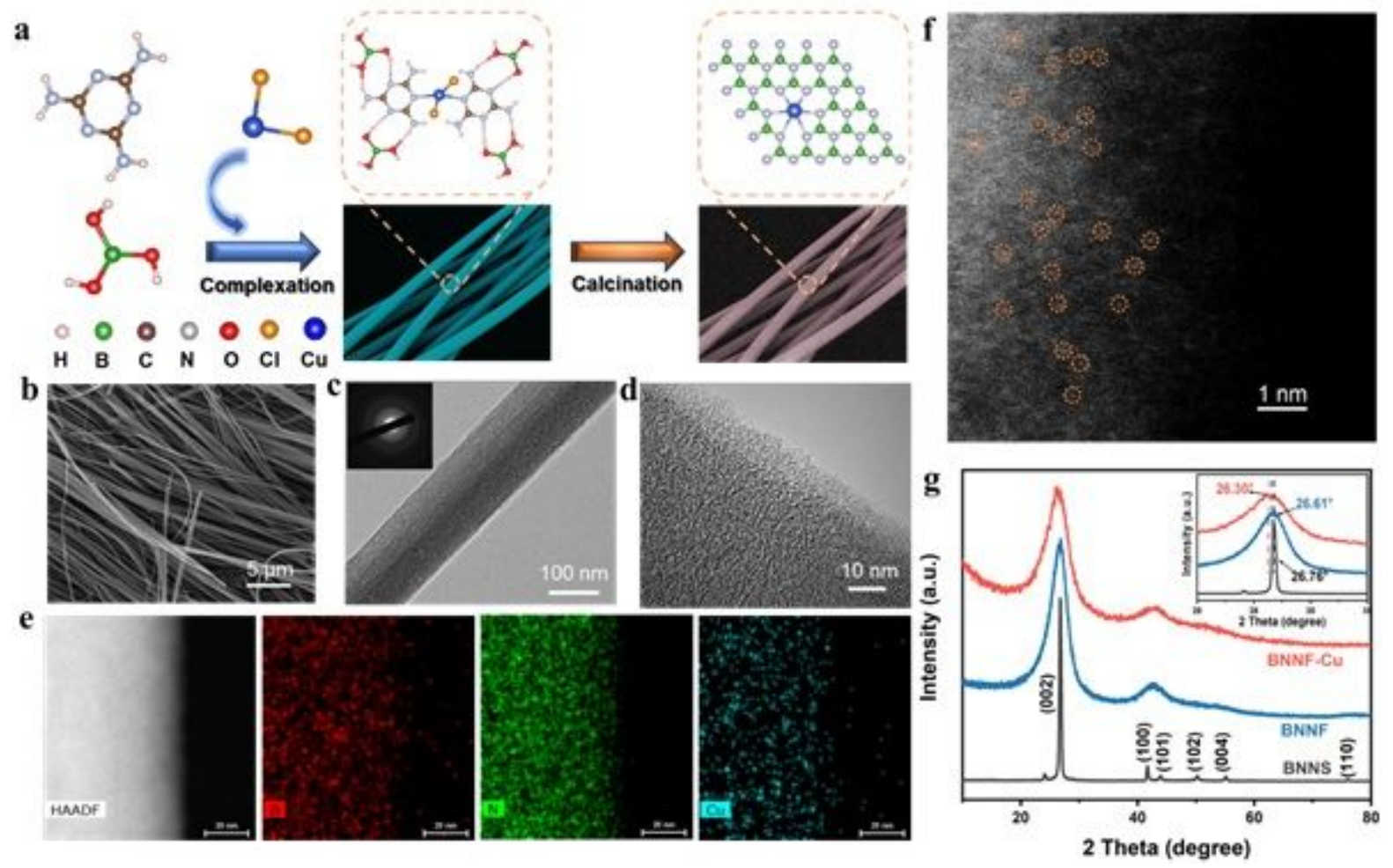

\section{Figure 1}

a Schematic diagram of the BNNF-Cu preparation process. b FE-SEM image $c$ typical TEM (inset SAED pattern), and d HRTEM images of BNNF-Cu. e HAADF mapping images and $f$ HAADF-STEM image of BNNF-Cu. g XRD patterns of BNNS, BNNF and BNNF-Cu. 

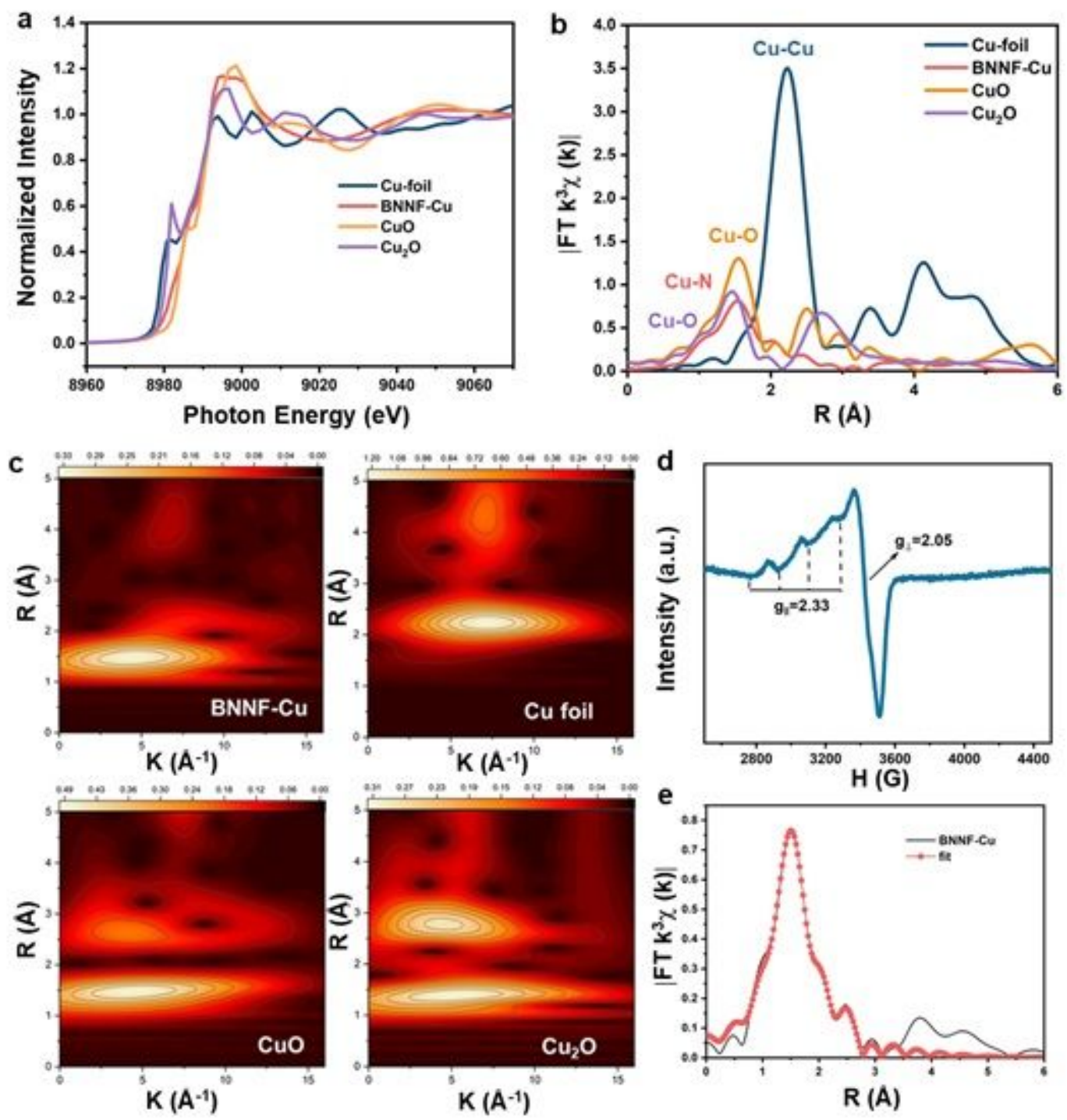

Figure 2

a Cu K-edge XANES and $b$ Fourier transform of the Cu K-edge EXAFS of BNNF-Cu and reference samples Cu foil, Cu2O and CuO. c Wavelet transforms for the EXAFS signals. d EPR spectrum of BNNF-Cu. e EXAFS fitting curve in $\mathrm{R}$ space. 

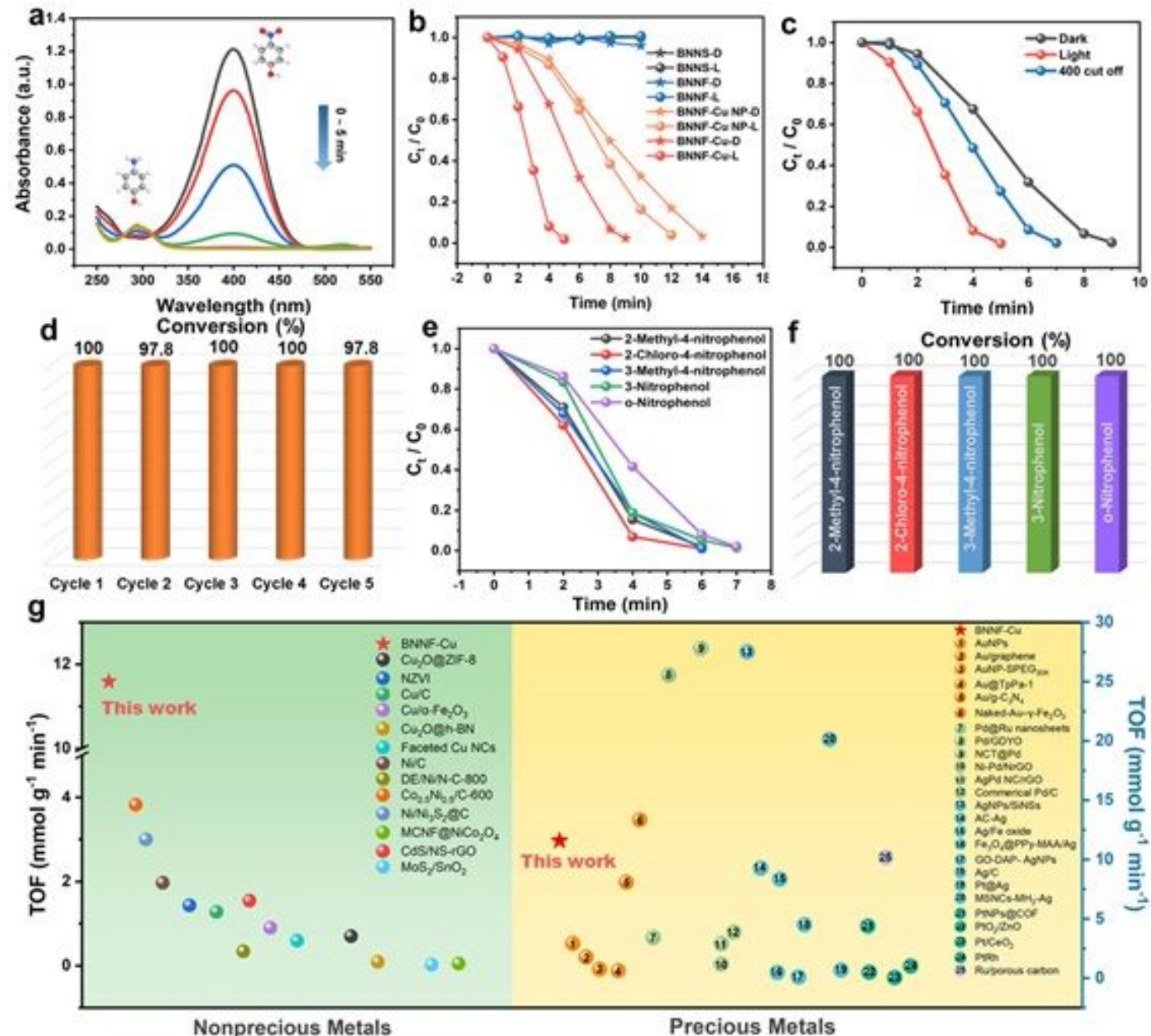

Figure 3

a Typical UV-vis absorption spectra of the reduction of PNP over BNNF-Cu. b Time-dependent PNP conversion of BNNS, BNNF, BNNF-Cu NP and BNNF-Cu under full spectrum or in darkness. c Timedependent PNP conversion of BNNF-Cu under full spectrum light, the light with a $400 \mathrm{~nm}$ filter and darkness. d Reusability of BNNF-Cu for reduction of PNP. e Conversion rates of the reduction of other nitroaromatic chemicals. $g$ Comparison of the catalytic activities of some reported catalysts for PNP reduction. The TOF is the number of reactant molecules that $1 \mathrm{~g}$ of metal can convert into products in per minutes (the unit of TOF is mmol g-1 min-1)
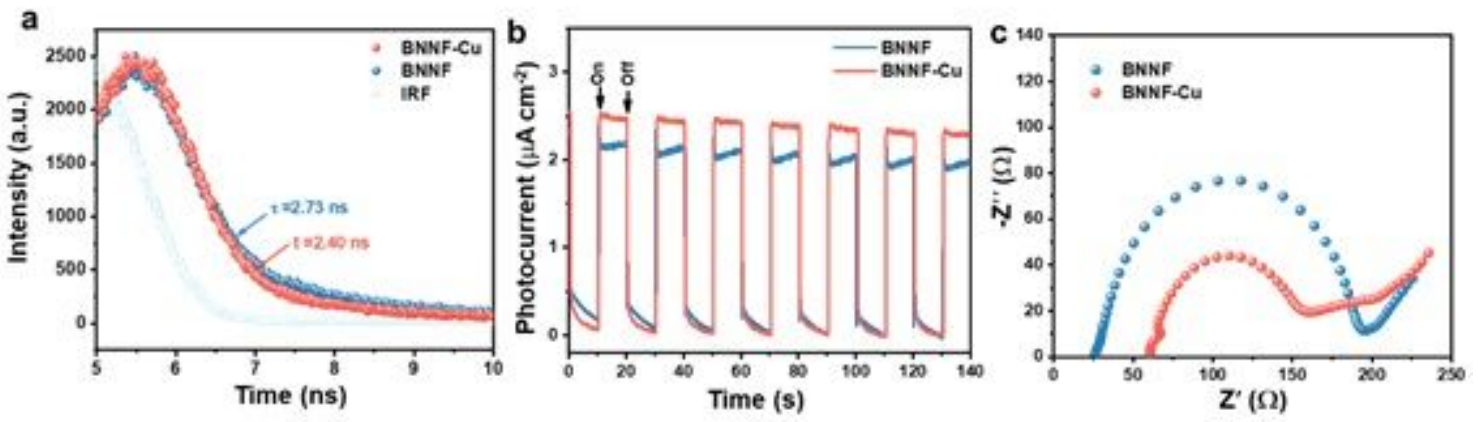
Figure 4

a TRPL spectra b Photocurrent response under irradiation and c Electrochemical impedance spectra of BNNF and BNNF-Cu.

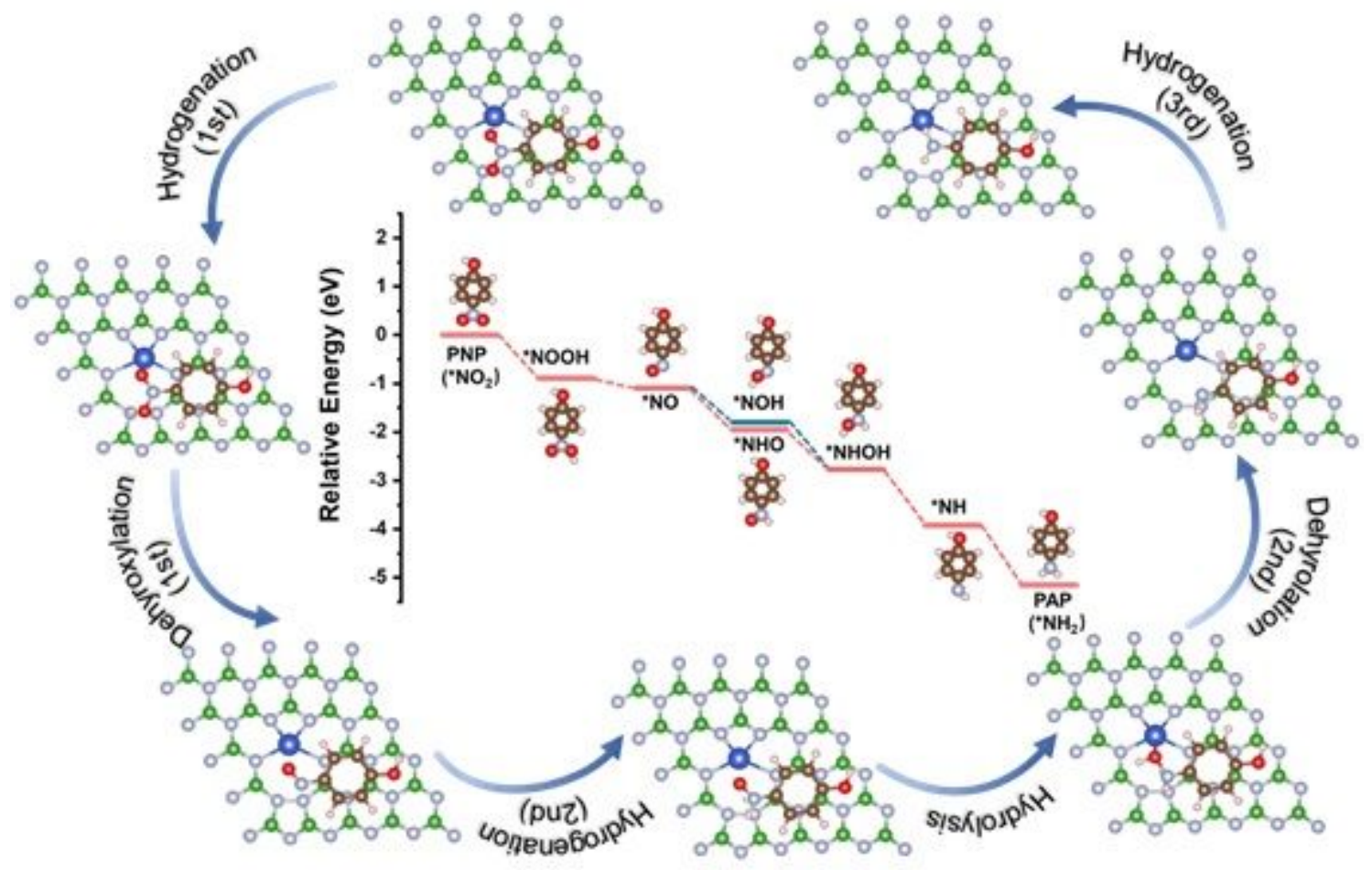

Figure 5

Schematic diagram of the reaction pathway for reduction of PNP to PAP over BNNF-Cu. The inset shows the free-energy diagram of PNP reduction of BNNF-Cu.

\section{Supplementary Files}

This is a list of supplementary files associated with this preprint. Click to download.

- Supplementaryinformation.docx 